\title{
Uso de Internet en el ámbito académico universitario
}

\section{Internet use in the field of academic university}

\author{
Moisés Ramírez Hernández ${ }^{1}$, Guadalupe Aurora Maldonado Berea ${ }^{2}$, Verónica Marín Díaz ${ }^{3}$ \\ ${ }^{1}$ Universidad Veracruzana, México (moshernandez@gmail.com) \\ ${ }^{2}$ Dirección de Innovación Educativa, Universidad Veracruzana, México (gumaldonado@uv.mx) \\ ${ }^{3}$ Facultad de Ciencias de la Educación, Universidad de Córdoba, España (ed1madiv@uco.es)
}

\section{RESUMEN:}

Este artículo muestra un análisis sobre el uso de Internet en actividades académicas por parte del profesor universitario, como resultado de una investigación en proceso cuyo objetivo es identificar el uso que hace el profesor de las TIC en su proceso de enseñanza. Este estudio combina los enfoques positivista y fenomenológico a través de una metodología mixta. Los instrumentos utilizados para la recolección de la información fueron la encuesta y el grupo de discusión. La muestra fue de 44 profesores de la Licenciatura en Pedagogía de la Región Veracruz, de la Universidad Veracruzana (UV) en México. Los resultados apuntan que es conveniente seguir realizando investigaciones en esta área.

\section{PALABRAS CLAVE: INTERNET, PROFESORES, EDUCACIÓN SUPERIOR, EXPERIENCIA EN LA ENSEÑANZA.}

\footnotetext{
ABSTRACT:

This paper presents an analysis of the use of internet in academic activities by the university professor, as a result of ongoing research aimed at identifying the use made Professor of ICT in their teaching process. This study combines the positivist and phenomenological approaches through a mixed methodology. The instruments used for data collection were the survey and focus group. The sample was 44 teachers in the Degree in Education at the Veracruz region of the Universidad Veracruzana (UV) in Mexico. The results suggest that it is appropriate to continue with these research type.
}

$\begin{array}{lrr}\text { KEYWORDS: } & \text { INTERNET, } & \text { TEACHERS, } \\ \text { HIGHER } & \text { EDUCATION, } & \text { TEACHING } \\ \text { EXPERIENCE. } & & \end{array}$

\section{INTRODUCCIÓN}

A lo largo de los últimos años, el ámbito de actuación de las Tecnologías de la Información y de la Comunicación (TIC) ha ido creciendo de manera vertiginosa, viendo su estela de impacto en áreas como la economía, la política, lo social o lo cultural, influyendo incluso en el ámbito educativo; es sorprendente, pues, la celeridad con que estas han modificado nuestro ritmo de vida, la manera en que nos comunicamos, así como también la forma de trabajar, decidir y pensar (Toffler, 1980; Prensky, 2001; Castell, 2002; Perrenoud 2004; Marín y Romero, 2009; Cabero, 2015).

En lo que se refiere al ámbito educativo, y más concretamente el nivel universitario, no puede pasar por alto lo que sucede en el mundo actual. En este sentido, Maldonado (2012) señala que la inclusión de las TIC en la docencia universitaria ha influido significativamente en cómo las instituciones de enseñanza superior han actualizado su propuesta formativa, el desarrollo de la docencia universitaria y los nuevos significados en el rol del docente y estudiante.

Asimismo, supone el incremento de las oportunidades de acceso al aprendizaje continuo, además de posibilitar que este sea flexible e interactivo, tomando como punto relevante el uso del Internet y sus respectivos servicios que ofrece.

Derivado de esto, la presente investigación se centra en la forma en cómo el profesorado 
aprehende y realiza el uso de las TIC y de Internet, por considerársele uno de los elementos con mayor importancia en el proceso de enseñanza y el aprendizaje, además que él juega un papel muy importante en el empleo que se haga o no de las TIC en el aula, y de lo que no podemos escapar es que "la era del ciberespacio está aquí y muchos maestros no se han percatado, peor aún, algunos rechazan a priori el uso de las Tecnologías de la Información y de la Comunicación (TIC) en sus actividades docentes" (Acuña, 2012, p. 9).

\subsection{Planteamiento del problema o tema objeto de estudio}

El surgimiento de las TIC han generado un cambio en las formas y prácticas docentes en la Educación Superior, debido a la prerrogativa de estudiar de qué manera influyen en esta, sin perder de vista la innovación educativa requerida tanto a este nivel educativo como a los docentes (Area, 2002), su pertinencia curricular (Díaz-Barriga, 2010), las técnicas didácticas empleadas (García-Valcárcel y Hernández, 2013), así como su impacto en la práctica docente y el aprendizaje (Barberà, 2008; Cabero, 2014; Marín, 2004; Marquès, 2007).

Este desarrollo ha influido de manera significativa en el rol del docente y del estudiante; de ahí la necesidad de documentar de manera continua los procesos y actos formativos de carácter permanente $\mathrm{y} / \mathrm{o}$ continuo de los profesores en primera instancia, y de la propia institución en segunda, así como de los estudiantes, receptores estos de las mejoras que los otros dos ámbitos realicen (Tascón, 2002).

En este sentido, se considera a la educación como el medio a través del cual los sujetos se preparan y se forman, con el fin de dar respuesta a necesidades sociales, tanto reales como potenciales (Morín, 1999), de ahí su carácter de "motor de cambio, progreso y cohesión social" (Area, 2002, p. 66).

Es por eso que las instituciones de educación superior (IES), intentan impulsar la calidad de la educación, así como el uso de las TIC atendiendo al cumplimiento de estándares que deben poseer tanto las IES como los docentes y sus estudiantes, entre los cuales se pueden mencionar el International Society for Technology in Education (ISTE, 2008) y la United Nations Educational, Scientific and Cultural Organization (UNESCO, 2011).

En este sentido, el concepto de calidad se vincula a la mejora de la educación, en donde Litwin (1995) asocia la innovación de la implementación de las TIC en el aula, a través de una nueva conceptualización de la enseñanza establecida en los diseños curriculares.

A partir de estas exigencias, las IES han adquirido un compromiso con la modernización educativa, incorporando las TIC en sus procesos de enseñanza y aprendizaje, al ser considerados un tema crucial, producido por su alta presencia en la vida de los individuos.

Estas circunstancias han producido que en la comunidad educativa se esté cuestionando acerca del uso que da el profesorado a las TIC, la repercusión de su implementación en el proceso de enseñanza y la forma en cómo las están incorporando a sus clases (Zabalza, 2009; GarcíaValcárcel, 2009, Marín, 2006; Díaz-Barriga, 2010), lo que conlleva a una inherente necesidad de investigar cómo el profesor hace uso o no de las TIC en el proceso de enseñanza.

En lo que se refiere a nuestro objeto de estudio, se considera que no existe discusión suficiente sobre lo que están haciendo los profesores en lo que respecta a la inclusión de las TIC en su dinámica diaria.

De ahí el interés de llevar a cabo este estudio, ya que a pesar que la educación universitaria es un tema muy estudiado tanto en México como en otros países, se ha identificado que la docencia ha sido un tema poco explorado y poco valorado, a pesar de que, como menciona Clark (1983), es una labor predominante de todo sistema de educación superior.

En este sentido, resulta necesario mostrar si la formación del profesorado guarda una correspondencia con el uso y percepción académica que hagan y tengan los profesores frente a las TIC y el uso de Internet, y con esto desarrollar nuevas metodologías didácticas, estrategias de enseñanza e innovaciones educativas.

\section{ANTECEDENTES Y FUNDAMENTACIÓN TEÓRICA}

Las IES se caracterizan por la creación y asimilación de conocimientos en sus desarrollos de cada momento, y del pensamiento crítico en los debates intelectuales de cada época. En donde, además, se originan y conforman los proyectos políticos y los grupos que los ponen en acción en todo el país y en cada estado (Cazes y Delgado, 2003).

Bajo este marco de referencia se puede identificar que las IES evolucionan a través del tiempo y del espacio, lo que nos lleva a pensar en cómo la práctica docente se ha posicionado en un 
determinado momento, y que por diversas circunstancias no ha trascendido más allá de una etapa en particular, pero en algunos casos, sí han ido transitando desde un interés personal e institucional en pro de mejorar su quehacer docente al emplear las TIC, que las toman como punto de transformación e innovación de la enseñanza.

Tal situación, en el caso de México, se puede mostrar a bajo la perspectiva del proceso sociohistórico de la tecnologización de la educación, a fin de tener una visión de la transformación y manejo de los recursos utilizados en la práctica educativa.

Rojas (2013) comenta con respecto a este tema que existen cuatro etapas, cuyas características generales son:

- Primer período, denominado "la maquinización de la enseñanza en el México decimonónico". Se caracteriza por el papel predominante de las máquinas y la expansión de la industrialización. La práctica educativa, es decir, la enseñanza no estuvo ajena a ello, pues los recursos utilizados (el aritmómetro, la pizarra calcante, las obritas, las cartillas y máquinas para "enseñar a escribir la letra inglesa", el silabario mecánico, las cartas geográficas y la caja enciclopédica para la enseñanza intuitiva) fueron invenciones del profesorado para la enseñanza de las ciencias y las artes en el nivel primario.

- Segundo periodo, nombrado como "la radio educativa en el México revolucionario". La incorporación de este recurso como medio educativo, se inicia con la estación mexicana CZF-XFX (1924-1937) fundada como emisora oficial de la SEP, cuya programación hacía énfasis en la educación rural, canto coral y gimnasia, dirigidos principalmente a los maestros rurales a modo de actualización docente; con este recurso se sentó un precedente en la incorporación de los medios de comunicación masiva en el ámbito educativo.

- Tercer período, identificado como la tecnología educativa en el México moderno de la segunda mitad del siglo XX. Los discursos oficiales en articulación con las políticas educativas giran en torno a la presencia de las tecnologías como estrategia para solucionar los problemas de cobertura, permanencia y atención del rezago educativo; además, la creación de dependencias oficiales (Dirección General de Televisión Educativa), redes de comunicación vía satélite (EDUSAT), las propuestas educativas (secundaria digital, videoteca educativa de las Américas, televisión educativa) y servicios educativos diversos. También se muestra un énfasis en la comunicación en el nivel universitario.

- Cuarto período, determinado como "el nuevo protagonismo tecnológico y la transposición didáctica del siglo XXI". Según los autores se hace énfasis al protagonismo tecnológico, las políticas, estrategias y acciones de organismos internacionales y nacionales, el uso de las TIC como propuesta globalizante y como instrumentación didáctica para los procesos educativos. En este periodo los recursos más destacados son: aula virtual, wiki, entornos virtuales de aprendizaje, plataformas Moodle, Enciclopedia, docente holográfico, videoconferencia, repositorios, mensajería electrónica, hipertexto y multimedia.

A través de estos períodos se pueden identificar las particularidades de los materiales, recursos e instrumentos que se incorporaron a los procesos de enseñanza $\mathrm{y}$, por lo tanto, al quehacer docente. En base en esta situación, se puede observar que las innovaciones tecnológicas de cada período influyeron de forma significativa en el proceso educativo, y lo hacen con mayor trascendencia aún hoy en día, incentivando la generación de políticas educativas relacionadas con las TIC en el sistema de educación superior.

México, en línea con esto, busca lograr una educación de calidad, que es una de las principales metas establecidas en su Plan Nacional de Desarrollo (PND) 2013-2018. En donde se resalta, entre otros aspectos, que la ciencia y la tecnología son puntos clave para alcanzar la sociedad del conocimiento, áreas caracterizadas por un bajo nivel de inversión en el país.

También señala que las habilidades que se requieren para tener éxito en el ámbito laboral han cambiado, pues la mayor parte de la información que existe es gracias a Internet, por lo que se requiere de ciudadanos que estén en condiciones de manejar e interpretar la información para poder generar conocimiento.

En el ánimo de alcanzar estas metas nacionales, el PND 2013-2018 específicamente en la meta de "Calidad en la Educación", con relación a la infraestructura, capacitación docente e implementación de las TIC, destaca las siguientes estrategias:

- Establecer un sistema de profesionalización docente que promueva la formación, selección, 
actualización y evaluación del personal docente y de apoyo técnico-pedagógico.

- Modernizar la infraestructura y el equipamiento de los recursos educativos.

- Promover la incorporación de las nuevas tecnologías de la información y comunicación en el proceso de enseñanza- aprendizaje.

Alineado con el PND se crea la Estrategia Digital para Todos (EDT), donde se establecen acciones y políticas para acercar las TIC a la población, buscando una inclusión digital y ciudadanos más informados. Mediante este plan de acción se busca insertar a México en la sociedad de la Información y del Conocimiento.

Enmarcada en esta misma línea, la Universidad Veracruzana (UV) establece sus políticas universitarias relacionadas con la habilitación, capacitación y formación de la planta académica; infraestructura tecnológica; evaluación y acreditación de los programas educativos $\mathrm{y}$, finalmente, uso de las TIC en los procesos de enseñanza-aprendizaje, a través de documentos como el Plan General de Desarrollo 2025, el Plan Estratégico de Tecnologías de la Información y Comunicación y el Programa de Trabajo Estratégico 2013-2017 “Tradición e Innovación”, con el fin de mantener su protagonismo y su búsqueda del desarrollo.

Encontrando como elemento importante del cambio el fortalecimiento de la capacidad académica, a través de programas orientados a la actualización y habilitación del personal docente (Universidad Veracruzana, 2014). Es por ello que, a través de la Dirección General de Desarrollo Académico e Innovación Educativa (DGDAIE), se han implementado cursos de formación tecnológica para la formación y fortalecimiento de sus docentes.

Situación que se destaca a partir de lo que Acuña (2012, p. 16) manifiesta, "el desarrollo tecnológico plantea nuevas propuestas educativas centradas en el alumno, por lo que urge la formación del profesorado en esta área, pues -además- juega un papel fundamental en la educación en los medios".

Es por ello que, como parte de la redefinición de su pertinencia social, en 1999 la UV puso en marcha el Modelo Educativo Integral y Flexible (MEIF), que está centrado en la formación integral de los estudiantes mediante un currículo flexible, donde las actividades de aprendizaje procuran cubrir los requerimientos del programa como las características del estudiante, y en donde las TIC han ido adquiriendo con el paso del tiempo un lugar cada vez más protagónico.
Y en el caso del objeto de estudio, los docentes de la Facultad de Pedagogía-Veracruz, cuentan con cierta infraestructura y equipamiento tecnológico, lo que hace evidente el acceso a estos recursos. Sin embargo, se hace necesario identificar de qué manera se han ido incorporando a los procesos de enseñanza de esta disciplina.

Con base en este contexto, se destaca la preocupación latente que ha existido por atender de manera adecuada el uso de las TIC en el proceso educativo, en atención especial al desarrollo de las competencias o saberes digitales que el profesor necesita hoy en día para llevar a cabo su rol como facilitador y gesto del aprendizaje.

En este sentido, para Ramírez-Martinell y Maldonado (2014), "las Tecnologías de la Información y Comunicación (TIC) han hecho evidente la necesidad de revisar y estudiar los enfoques en la forma de enseñar y aprender en el contexto de la educación superior" (p. 19).

Asimismo, Crovi (2009) destaca que las TIC han ido tomando cada vez mayor relevancia desde finales de la década de los setenta, a partir de los descubrimientos tecnológicos, que se denominarían posteriormente como tecnologías digitales, tecnologías de información o tecnologías de información y comunicación.

De ahí la importancia de mostrar la incorporación de las TIC y el uso de Internet en el proceso de enseñanza universitaria $\mathrm{y}$, por lo tanto, su influencia en el quehacer docente.

Como muchas de las herramientas que conforman el universo de las TIC, Internet o la autopista de la información surgió como respuesta a una necesidad de comunicación, cuyo fin era que se llevara a cabo esta de forma más segura y robusta en las instituciones dentro del campo militar, empresarial y económico (Maldonado, 2012).

$\mathrm{El}$ acceso abierto y gratuito a documentos básicos ha sido la clave para el rápido crecimiento de Internet, aunado al hecho de que "la comunidad investigadora universitaria promovieron la tradición académica de publicar ideas y resultados de forma abierta" (Leiner, Cerf, Clark, Kahn, Kleinrock, Lynch, Postel, Roberts y Wolff, 2012).

En este sentido se comprende a las TIC como el conjunto de artefactos y aplicaciones electrónicas a través de los cuales los usuarios se conectan, producen, seleccionan e intercambian información y conocimientos, principalmente vía Internet (Alvarado, 2005 citado por Torres, Barona, Zúñiga y Soberanes, 2012).

En la sociedad de la información y del conocimiento es indispensable comprender el 
avance que tienen los centros universitarios en el cumplimiento de las metas de adopción de tecnologías avanzadas, y es que la educación para una sociedad del conocimiento y globalizada considera que el uso de estas tecnologías en los entornos y procesos de aprendizaje es un elemento consustancial.

Por lo antepuesto, han surgido diversas teorías, o corrientes pedagógicas, que datan el uso de estos recursos para la educación y en mayor medida en los procesos de enseñanza y en la interacción profesor-estudiante; destacando entre ellas:

- El conectivismo, se ha convertido en una de las teorías más abordadas en el ámbito de las TIC en la educación: De acuerdo con Pérez (2012) "el crecimiento y el grado de complejidad del conocimiento implica que la capacidad de aprender resida en las conexiones que formamos con otras personas y grupos, generalmente mediadas y facilitadas por la tecnología digital" (p. 104); el mismo autor se refiere a esta como "un aprendizaje educativo como un proceso de conexión de fuentes de información y nodos especializados de conocimiento" (p. 110).

- El constructivismo. Aldama (2004) menciona que en el constructivismo el profesor reduce su nivel de autoridad, para que el estudiante desarrolle su autonomía en el aprendizaje y así evitar la dependencia moral e intelectual. Es aquí donde resulta importante que el docente explore por sí mismo, descubra y construya su nueva forma de ser y actuar en el proceso didáctico, promoviendo la construcción del conocimiento. En este caso, mediado por el uso de las TIC. En congruencia con lo anterior, aun cuando el proceso de enseñanza-aprendizaje esté bajo "un modelo pedagógico tradicional, el uso de las nuevas tecnologías tendría efectos beneficiosos sobre aquellas tareas que suponen identificar y recolectar información y en la mejor hipótesis posible, podría facilitar la enseñanza de cómo transformar esa información en nuevo conocimiento" (Brunner, 2003, p. 130).

Es a partir del constructivismo, que este estudio busca identificar cuál es el uso que los profesores de la Facultad de Pedagogía Veracruz hacen de la tecnología y cómo favorece a la construcción del conocimiento de los aprendices. Es por ello que se hace evidente la formación en TIC del profesorado universitario.
En este sentido, se toma el concepto de uso, como la práctica cotidiana y el empleo de recursos materiales y digitales relevantes para el proceso educativo (Crovi, 2009), y particularizándolo al proceso de enseñanza, se comprende como la manera en que el profesor emplea herramientas, recursos y estrategias mediadas por las TIC en su metodología de trabajo para propiciar la construcción de conocimientos en sus educandos.

Con relación en lo expuesto sobre el empleo de las TIC con fines educativos y su incorporación en los procesos de enseñanza aprendizaje, Manso, Pérez, Libedinsky, Light y Garzón (2014) proponen cinco pasos para la planificación de actividades con TIC los cuales se enumeran a continuación:

- Desarrollar los objetivos de aprendizaje

- Decidir las estrategias de enseñanza que se van a utilizar.

- Diseñar o seleccionar las actividades de aprendizaje.

- Seleccionar las estrategias de evaluación

- Seleccionar y articular las herramientas o los recursos TIC para las actividades.

$\mathrm{Si}$ bien, los pasos anteriores solo son una recomendación, pues cada profesor puede decidir la manera de cómo incorporar estos recursos a la educación.

\section{DISEÑO Y METODOLOGÍA}

Derivado de la naturaleza de la investigación y con la finalidad de responder a las interrogantes y cumplir con los objetivos planteados en el estudio, se determinó enlazar los paradigmas cuantitativo y cualitativo a través de una metodología mixta, utilizando un diseño no experimental con un corte transversal-descriptivo y fenomenológico (Hernández, Fernández y Baptista, 2010). Para el caso del presente artículo, se hace énfasis en solo tres secciones de los resultados obtenidos desde el enfoque cuantitativo, los cuales se explican en la parte de resultados.

La población de estudio fueron los profesores de la Licenciatura en Pedagogía, cuyo escenario es la Facultad de Pedagogía, Región Veracruz, de la Universidad Veracruzana, institución de educación superior pública con mayor impacto en el sureste de la República Mexicana.

De las técnicas e instrumentos para la recolección de la información desde el enfoque cuantitativo se utilizó la encuesta. 
Desde la perspectiva cuantitativa se aplicó un cuestionario diseñado en el proyecto "Brecha digital entre estudiantes y profesores de la Universidad Veracruzana: Capital cultural; trayectorias escolares y desempeño académico; y grado de apropiación tecnológica" (Ramírez-Martinell, Casillas y Ojeda, 2013), que busca identificar esencialmente el uso de TIC en estudiantes y profesores.

Dicho instrumento contiene 44 preguntas de diferente formato, cerradas, tipo Likert, dicotómicas y abiertas. Se encuentra estructurado por 11 secciones: 1. Datos de identificación, 2. Socioeconómico, 3. Afinidad tecnológica, 4. Literacidad digital, 5. Ciudadanía digital, 6. Comunicación, socialización y colaboración, 7. Programas y sistemas de información relativos a su área de conocimiento, 8. Dispositivos, 9. Archivos, 10. Sotfware de oficina, 11. Creación y manipulación de contenido multimedia.

Estadísticamente se trató de realizar un censo, procurando trabajar con los 50 profesores que integraban la planta académica, es decir, sin realizar algún tipo de exclusión o muestreo. Con el fin de encuestar a toda la población la aplicación se realizó en dos momentos. En el periodo comprendido de febrero-julio 2014 se aplicaron 22 cuestionarios y durante el periodo agosto 2014 a enero 2015 se contestaron 22 cuestionarios más. El resultado final fueron 44 cuestionarios contestados por el profesorado, es decir, el $88 \%$, porcentaje representativo para poder llevar a cabo el estudio.

De los sujetos encuestados el $43.18 \%$ corresponde al género masculino y el $56.82 \%$ al femenino. De acuerdo a su tipo de contratación en su mayoría son profesores de tiempo completo
(34.09\%) y de horas base por asignatura (36.36\%), gran cantidad de ellos cuentan con grado académico de doctorado (25\%) y maestría (43.18\%).

Para el procesamiento de la información obtenida, se empleó el Statistical Package for the Social Sciences (SPSS) versión 22 para Windows. Con la ayuda de este programa se realizó una distribución de frecuencias.

\section{RESULTADOS}

En este artículo se exponen resultados preliminares del estudio de tres secciones del instrumento: socioeconómico, afinidad tecnológica y literacidad digital.

\subsection{Nivel socioeconómico de los profesores}

En este primer apartado del cuestionario se buscó identificar los dispositivos digitales que posee un profesor, ya sean propios o proporcionados por la institución, así como el acceso a Internet, servicios y recursos con los que cuenta.

Cantidad de dispositivos que poseen los profesores, tanto Apple como de otras marcas.

En la tabla 1 se puede observar que solo 1 profesor $(2.3 \%)$ dijo tener de la marca Apple computadora de escritorio propia, laptop propia o familiar, computadora o laptop asignada por la UV, y computadora o laptop comprada con recursos de proyectos externos. En la misma línea 2 profesores $(4.5 \%)$ dijeron tener una tableta y 8 de ellos (18.2\%) dijo tener un celular con conexión a Internet de la misma marca.

Tabla 1. Cantidad de dispositivos tanto Apple como de otras marcas (\%)

\begin{tabular}{|c|c|c|c|c|c|c|c|}
\hline \multirow[t]{2}{*}{ Dispositivos } & \multicolumn{4}{|c|}{ Numero de dispositivos de otras marcas } & \multicolumn{3}{|c|}{$\begin{array}{l}\text { Número de dispositivos de } \\
\text { marca Apple }\end{array}$} \\
\hline & 0 & 1 & 2 & 3 & 0 & 1 & 2 \\
\hline Computadora de escritorio propia & 29.5 & 65.9 & 4.5 & 0 & 97.7 & 2.3 & 0 \\
\hline Laptop propia(o familiar) & 9.1 & 75.0 & 13.6 & 2.3 & 95.5 & 2.3 & 2.31 \\
\hline $\begin{array}{l}\text { Computadora o laptop asignada por la } \\
\text { UV(en el centro de cómputo u oficina) }\end{array}$ & 61.4 & 31.8 & 6.8 & 0 & 97.7 & 2.3 & 0 \\
\hline $\begin{array}{l}\text { Computadora o laptop comprada con } \\
\text { recursos de proyectos externos (PROMEP, } \\
\text { CONACYT u otros) }\end{array}$ & 86.4 & 13.6 & 0 & 0 & 97.7 & 2.3 & 0 \\
\hline Tableta & 70.5 & 27.3 & 2.3 & 0 & 95.5 & 4.5 & 0 \\
\hline Teléfono celular con conexión a Internet & 25.0 & 72.7 & 2.3 & 0 & 81.8 & 18.2 & 0 \\
\hline
\end{tabular}

Por otro lado, sumando a los profesores que mencionaron tener uno, dos o tres equipos tecnológicos, 40 de ellos (90.9\%) poseen una laptop propia de otras marcas; mientras que más del $70 \%$ cuentan con computadora de escritorio propia y teléfono celular con conexión a Internet. De manera general los profesores de la Facultad de Pedagogía prefieren dispositivos de otras marcas e invierten muy poco en dispositivos tecnológicos marca Apple.

Esta información es muy importante, porque de esta manera tenemos una idea general de los 
dispositivos con los que cuenta el profesor y utiliza para conectarse a Internet.

Percepción sobre el uso de Internet a través de dispositivos para realizar actividades académicas

Con la finalidad de operativizar la presentación de los datos, la escala de respuesta se ha recategorizado aunando de un lado la opción Muy de acuerdo (4) con De acuerdo (3), de otro En desacuerdo (1) y Muy en desacuerdo (0).

Como se refleja en la tabla 2, más del $80 \%$ de los docentes consideran que poseer una computadora o laptop, además de tener acceso a Internet, es indispensable para sus actividades académicas; frente a un $7.8 \%$ que piensa que no es necesario. Los datos también reflejan un porcentaje similar en las opiniones que tienen los profesores sobre lo indispensable que es poseer una tableta para las actividades académicas, ya que el $45.5 \%$ mantienen una valorización positiva, mientras que un $43.1 \%$ piensa lo contrario. Por otro lado, el $65.9 \%$ señala que poseer un celular con conexión a Internet es indispensable para sus actividades académicas.

Finalmente, otro dato importante es que el $79.6 \%$ de los profesores creen que los recursos tecnológicos no son un símbolo de prestigio.

Tabla 2. Uso de internet en actividades académicas (\%)

\begin{tabular}{ccccccc}
\hline & 4 & 3 & 2 & 1 & 0 & NC \\
\hline $\begin{array}{c}\text { Poseer una laptop, computadora, tableta o celular con } \\
\text { conexión a Internet es un símbolo de prestigio }\end{array}$ & 2.3 & 13.6 & 4.5 & 52.3 & 27.3 & 0 \\
$\begin{array}{c}\text { Poseer una computadora o laptop es indispensable para mis } \\
\text { actividades académicas }\end{array}$ & 63.6 & 25.0 & 4.5 & 2.3 & 4.5 & 0 \\
$\begin{array}{c}\text { Poseer una tableta es indispensable para mis actividades } \\
\text { académicas }\end{array}$ & 25.0 & 20.5 & 11.4 & 29.5 & 13.6 & 0 \\
$\begin{array}{c}\text { Poseer un celular con conexión a Internet es indispensable } \\
\text { para mis actividades académicas }\end{array}$ & 40.9 & 25.0 & 6.8 & 15.9 & 9.1 & 2.3 \\
$\begin{array}{c}\text { Tener acceso a Internet es indispensable para mis actividades } \\
\text { académicas }\end{array}$ & 65.9 & 22.7 & 4.5 & 2.3 & 4.5 & 0 \\
\hline \begin{tabular}{c} 
acalo \\
\hline
\end{tabular} & & & & &
\end{tabular}

Frecuencia y tipo de conexión a Internet para fines académicos y no académicos

En esta sección se pretendió identificar el tipo de conexión y la frecuencia con la que navega en Internet para fines académicos. En la tabla 3 se visualiza que más del $50 \%$ de los profesores prefieren conectarse "siempre" en la red de la Universidad para realizar actividades académicas. Si recategorizamos las opciones de respuesta "Siempre, frecuentemente y algunas veces", encontramos que el $90.9 \%$ navega a través de la red de la Universidad, el $81.9 \%$ mediante servicio de Internet en casa, el $72.7 \%$ con la conexión de un celular con plan, y muy por debajo de estos porcentajes el $18.2 \%$ por medio de un dispositivo de banda ancha.

Se logra apreciar a su vez, que el 77.3\% "nunca" se conectan a través de un dispositivo de banda ancha para sus actividades académicas, en posición extrema al $11.4 \%$ que mencionan siempre utilizarlo.

Tabla 3. Frecuencia en el uso del Internet con fines académicos (\%)

\begin{tabular}{ccccccc}
\hline & Siempre & Frecuentemente & Algunas veces & Casi nunca & Nunca & $\begin{array}{c}\text { No } \\
\text { contesto }\end{array}$ \\
\hline Servicio Internet en casa & 45.5 & 34.1 & 2.3 & 2.3 & 13.6 & 2.3 \\
En la red de la Universidad & 56.8 & 20.5 & 13.6 & 2.3 & 6.8 & 0 \\
A través de mi celular con plan & 31.8 & 25.0 & 15.9 & 4.5 & 22.7 & 0 \\
$\begin{array}{c}\text { A través de un dispositivo de } \\
\text { banda ancha (BAM) }\end{array}$ & 11.4 & 6.8 & 0 & 2.3 & 77.3 & 2.3 \\
\hline
\end{tabular}

De igual forma, se presenta la frecuencia con la que los profesores se conectan a través de los diferentes servicios con fines no académicos (ver tabla 4), aunque los datos reflejan un porcentaje igual en el uso de servicio de Internet en casa mostrando un $29.5 \%$ para los casos "siempre" y "nunca"; al recategorizar las columnas de "siempre "y "frecuentemente" se manifiesta que el 50\% de los profesores prefieren navegar en la red en casa para realizar actividades no académicas, el mismo porcentaje lo encontramos en la opción interactuar en Internet a través de su celular. Por otra parte, el $50 \%$ ("casi nunca" y "nunca") prefieren evitar 
conectarse en la red de la Universidad para dichas actividades.
Finalmente, el $70.5 \%$ de los profesores "nunca" navegan a través de un dispositivo de banda ancha, mientras que solo el $6.85 \%$ lo hace.

Tabla 4. Frecuencia en el uso del Internet con fines NO académicos.

\begin{tabular}{ccccccc}
\hline & Siempre & Frecuentemente & $\begin{array}{c}\text { Algunas } \\
\text { veces }\end{array}$ & Casi nunca & Nunca & $\begin{array}{c}\text { No } \\
\text { contesto }\end{array}$ \\
\hline Servicio Internet en casa & 29.5 & 20.5 & 9.1 & 6.8 & 29.5 & 4.5 \\
En la red de la Universidad & 18.2 & 11.4 & 11.4 & 13.6 & 36.4 & 9.1 \\
A través de mi celular con plan & 34.1 & 15.9 & 2.3 & 6.8 & 31.8 & 9.1 \\
A través de un dispositivo de banda & 6.8 & 9.1 & 0 & 4.5 & 70.5 & 9.1 \\
ancha (BAM) & & & & & & \\
\hline
\end{tabular}

\section{Inversión en servicios de Internet}

De manera general, como se puede apreciar en la tabla 5, el $81.8 \%$ de los sujetos participantes en este estudio, invierte más en Internet en casa, en el teléfono $(75 \%)$ y en el pago de televisión por cable $(72.7 \%)$.

Tabla 5. Inversión en servicios TIC (\%)

\begin{tabular}{ccc}
\hline & $\mathrm{Si}$ & No \\
\hline Teléfono en casa & 75.0 & 25.0 \\
Televisión por cable & 72.7 & 27.3 \\
Internet en casa & 81.8 & 18.2 \\
Televisión bajo demanda & 13.6 & 86.4 \\
Teléfono celular en plan & 59.1 & 40.9 \\
Teléfono celular de prepago & 22.7 & 77.3 \\
\hline
\end{tabular}

\subsection{Afinidad tecnológica de los profesores}

A través de esta sección se busca descubrir o identificar las percepciones, valoraciones, actitudes por parte de los profesores en relación al uso y dominio de las TIC, prioritariamente en el ámbito académico.

Percepción sobre los resultados que genera el dominio de TIC en actividades académicas

Como se muestra en la tabla 5, la mayor parte de los profesores indican que están "Muy de acuerdo" y "De acuerdo" en que aquel que domina las TIC tiene mejores resultados en las diversas actividades académicas de docencia, gestión, tutoría, investigación y vinculación.

Tabla 5. TIC en actividades académicas (\%)

\begin{tabular}{ccccccc}
\hline & Muy de acuerdo & De acuerdo & Indeciso & En desacuerdo & $\begin{array}{c}\text { Muy en } \\
\text { desacuerdo }\end{array}$ & NC \\
\hline Docencia & 50 & 34.1 & 11.4 & 4.5 & 0 & 0 \\
Gestión & 45.5 & 45.5 & 4.5 & 4.5 & 0 & 0 \\
Tutoría & 43.2 & 31.8 & 13.6 & 11.4 & 0 & 0 \\
Investigación & 63.6 & 29.5 & 2.3 & 4.5 & 0 & 0 \\
Vinculación & 50 & 40.9 & 6.8 & 2.3 & 0 & 0 \\
Ejecución y/o creación & 43.2 & 40.9 & 9.1 & 2.3 & 2.3 & 2.3 \\
\hline
\end{tabular}

\section{Importancia de la conexión a Internet}

En la tabla 6, se puede observar que el $63.7 \%$ de los profesores mantienen una valoración positiva, al mencionar que estar conectado a Internet permanentemente es indispensable para la realización de sus actividades; más de la mitad (54.6\%) también piensa que navegar a través de la red es la mejor manera de encontrar información académica, el $72.7 \%$ de ellos piensa que es fundamental mantener un software actualizado en sus dispositivos digitales. Continuando con la línea de agrupación de ítems de respuesta, en lo que se refiere a la opción de respuesta Muy de acuerdo y De acuerdo, encontramos que más de la mitad $(52.3 \%)$ piensa que el celular con conexión a Internet es indispensable para su vida cotidiana; una gran parte, el $38.7 \%$ se sitúa en una postura contraria, además de un $9.1 \%$ que mantiene sus dudas que así sea. 
Tabla 6. Importancia de la conexión a Internet

\begin{tabular}{|c|c|c|c|c|c|}
\hline & $\begin{array}{l}\text { Muy de } \\
\text { acuerdo }\end{array}$ & $\begin{array}{c}\text { De } \\
\text { acuerdo }\end{array}$ & Indeciso & $\begin{array}{c}\text { En } \\
\text { desacuerdo }\end{array}$ & $\begin{array}{c}\text { Muy en } \\
\text { desacuerdo }\end{array}$ \\
\hline $\begin{array}{c}\text { Estar conectado a Internet permanentemente es } \\
\text { indispensable para mis actividades }\end{array}$ & 20.5 & 43.2 & 13.6 & 20.5 & 2.3 \\
\hline $\begin{array}{l}\text { La mejor manera de encontrar información académica es } \\
\text { a través de Internet }\end{array}$ & 11.4 & 43.2 & 11.4 & 31.8 & 2.3 \\
\hline $\begin{array}{l}\text { Es indispensable mantenerme actualizado con las } \\
\text { últimas versiones de software o dispositivos digitales }\end{array}$ & 34.1 & 38.6 & 13.6 & 13.6 & 0 \\
\hline $\begin{array}{l}\text { El celular con conexión a Internet es indispensable para } \\
\text { mi vida cotidiana }\end{array}$ & 18.2 & 34.1 & 9.1 & 36.4 & 2.3 \\
\hline
\end{tabular}

\subsection{Literacidad digital}

En esta sección principalmente se buscó identificar cómo accede el usuario a la información, y la forma en la que realiza las búsquedas.

Acciones que llevan a cabo los profesores al realizar búsquedas en Internet
En relación a las acciones que los profesores efectúan al hacer búsquedas por Internet, en general los resultados son muy altos en la mayoría de los rubros, tal y como se puede observar en la tabla 7 .

Tabla 7. Acciones al realizar búsqueda en Internet (\%)

\begin{tabular}{cccc}
\hline & Si & No & NC \\
\hline Realizo búsquedas avanzadas a través de los buscadores & 81.8 & 15.9 & 2.3 \\
Uso Google académico & 93.2 & 6.8 & 0 \\
Cuando hago una consulta en un buscador sé lo que estoy & 93.2 & 6.8 & 0 \\
buscando & & & \\
Utilizo palabras claves o representativas & 77.3 & 20.5 & 2.3 \\
Contrasto la información con diferentes fuentes & 90.9 & 9.1 & 0 \\
Reconozco fuentes fiables de información & 93.2 & 6.8 & 0 \\
Uso de operadores booleanos (and, or) & 34.1 & 63.6 & 2.3 \\
Empleo operadores de búsqueda (") & 52.3 & 45.5 & 2.3 \\
\hline
\end{tabular}

Un dato relevante es que si bien los profesores declaran realizar búsquedas avanzadas, un $63.6 \%$ menciona no utilizar operadores boléanos para potenciar su búsqueda. Por otra parte el empleo de operadores de búsqueda también se encuentra bastante limitado, pues un $45.5 \%$ no lo utiliza.

Acciones al elaborar un trabajo académico a través de Internet
Recategorizando las opciones de respuesta "Siempre" y "Frecuentemente", nos encontramos que un $97.7 \%$ de los profesores reconoce cuando la información es veraz y confiable, mientras que un $90.9 \%$ examina puntos de vista de diferentes autores e identifica argumentos importantes para construir conclusiones razonables de la información (ver tabla 8).

Tabla 8. Acciones al realizar trabajo académico a través de internet (\%)

\begin{tabular}{|c|c|c|c|c|c|}
\hline & Siempre & Frecuentemente & $\begin{array}{c}\text { Algunas } \\
\text { veces }\end{array}$ & Casi nunca & Nunca \\
\hline $\begin{array}{l}\text { Reconozco cuando la información a la que } \\
\text { accedo es veraz y confiable }\end{array}$ & 47.7 & 50 & 0 & 0 & 2.3 \\
\hline $\begin{array}{l}\text { Examino puntos de vista de diferentes autores, } \\
\text { incluso con los que no estoy de acuerdo }\end{array}$ & 47.7 & 43.2 & 4.5 & 2.3 & 2.3 \\
\hline $\begin{array}{l}\text { Identifico los argumentos importantes de la } \\
\text { información, lo destaco, evalúo y analizo para } \\
\text { después construir conclusiones razonables }\end{array}$ & 52.3 & 38.6 & 6.8 & 0 & 2.3 \\
\hline
\end{tabular}




\section{CONCLUSIONES Y DISCUSIÓN DE LOS RESULTADOS}

A partir de los resultados expuestos en el apartado anterior, podemos mencionar que el profesor de la Facultad de Pedagogía en su mayoría cuenta con al menos un dispositivo digital (computadora de escritorio, laptop, tableta o teléfono celular) para sus actividades académicas o cotidianas. Estos en su mayoría son de marcas diversas, no predominando en esta población la marca Apple, probablemente por los altos costos para su adquisición ya sea para uso personal o por la institución educativa.

Es interesante puntualizar que aunque hay una fuerte presencia de las computadoras portátiles, la de escritorio sigue teniendo presencia entre los usuarios.

En línea con lo anterior, el profesor muestra una valoración positiva al expresar que tener una computadora, laptop, tableta o celular con conexión a Internet es indispensable para poder realizar sus actividades académicas. Pero no consideran que sean un símbolo de prestigio, es decir, está de acuerdo que el uso de dispositivos y estar conectado a la red favorece en sus actividades académicas e incluso facilita la labor docente, pero no le da un status sobre sus pares o la comunidad universitaria.

Para sus actividades académicas, los profesores prefieren conectarse a través de la red institucional, esto es comprensible pues desde sus aulas o departamentos pueden realizar actividades de docencia, gestión, investigación, tutoría, vinculación y ejecución o creación, es decir todas las acciones que conlleva su práctica profesional.

Por otra parte, también mencionaron realizar actividades desde la conexión a Internet en casa, puede ser esto posible al realizar sus planeaciones didácticas. También mantienen conexión desde su celular con plan, lo que les permite tener más fácil acceso a la información, así como mantener comunicación con sus estudiantes y pares.

En sintonía con lo anterior, los datos también revelaron que el profesor hace una fuerte inversión en Internet en casa, pues independientemente de realizar actividades académicas, utiliza la conexión para sus actividades cotidianas.

Con el apoyo de Internet los profesores realizan acciones de literacidad digital, con mayor dominio el uso de Google Académico; además, distingue fuentes fiables de información al tener claro lo que está buscando, y contrasta la información con otras fuentes, lo que les permite ser analíticos y reflexivos en sus tareas. No obstante, se le dificulta hacer uso de operadores booleanos y de búsqueda.

El profesor argumenta reconocer información veraz y confiable, examina puntos de vista de diferentes autores, identifica argumentos importantes para poder construir conclusiones razonables.

Con toda esta reflexión, se destaca que el Internet es valorado positivamente pues ha facilitado el actuar humano y la actividad académica.

\section{PROSPECTIVA}

Esta investigación en primera instancia da un diagnóstico sobre el uso que hace el profesor universitario de las TIC. En este caso del uso de dispositivos digitales y la conexión a Internet como apoyo a sus actividades académicas. Este estudio es una evidencia clara para que la Universidad Veracruzana tenga nociones sobre el uso de la red institucional, y de manera particular el empleo de la red por parte del profesor de la Licenciatura en Pedagogía.

Es necesario continuar con estudios relacionados con la brecha digital, la formación docente, la apropiación tecnológica y en esta disciplina en particular, identificar la contraparte, es decir, el acceso y uso de los estudiantes universitarios.

\section{REFERENCIAS}

Acuña, A. (2012). El uso de las Tecnologías de la Información y la Comunicación (TIC) en la educación. México: ANUIES, Dirección de Medios Editoriales.

Aldama, G. (2004). Práctica docente para renovar el aprendizaje. México: Esfinge.

Area, M. (2002). La integración escolar de las nuevas tecnologías entre el deseo y la realidad. Organización y gestión educativa, 6, 14-18.

Barberà, E. (2008). Aprender e-Learning. Madrid: Paidós.

Brunner, J. J. (2003). Educación e Internet ¿La próxima revolución? Santiago: Fondo de Cultura Económica.

Cabero, J. (2014). La formación del profesorado en TIC: Modelo TPACK (conocimiento tecnológico, pedagógico y de contenido). Sevilla: Universidad de Sevilla.

Cabero, J. (2015). Reflexiones educativas sobre las tecnologías de la información y la comunicación (TIC). Tecnología, ciencia y educación, I, 19-27.

Castells, M. (2002). La era de la información. La sociedad red (Vol. I). México, D.F: Siglo XXI.

Cazes, M. D., y Delgado, W. R. (2003). Hacia una política de Estado para la Educación superior en México. México: Porrúa.

Clark, B. (1983). El sistema de educación Superior. Una visión comparativa de la organización académica. México: Nueva Imagen. 
Crovi, D. (2009). Acceso, uso y apropiación de las TIC en comunidades académicas. Diagnostico en la UNAM. México: UNAM y Plaza y Valdés.

Díaz-Barriga, A. (2010). TIC en el trabajo en el aula. Impacto en la planeación didáctica. Revista Iberoamericana de Educación Superior, 10(IV), 3-21.

García-Valcárcel, A. (2009). La incorporación de las TIC en la docencia universitaria: recursos para la formación del profesorado. Barcelona: Davinci Continental.

García-Valcárcel, A., y Hernández, A. (2013). Recursos tecnológicos para la enseñanza e innovación educativa. Madrid: Síntesis.

Hernández, R., Fernández, C., y Baptista, P. (2010). Metodología de la Investigación. México: McGrawHill.

ISTE (2008). ISTE Standards Teachers. Recuperado de http://www.iste.org/docs/pdfs/20-14_ISTE_StandardsT_PDF.pdf

Leiner, B., Cerf, V., Clark, D., Kahn, R., Kleinrock, L., Lynch, D., Postel, J., Roberts, L., y Wolff, S. (2012). Breve Historia de Internet. Recuperado 15 de octubre de 2015, a partir dede http://www.internetsociety.org/es/breve-historia-deinternet

Litwin, E. (1995). Tecnología educativa Política, historias, propuestas. Buenos Aires: Paidós.

Maldonado, G. A. (2012). Actitudes con respecto al uso de la plataforma tecnológica de teleformación Moodle: El caso de los estudiantes de la Facultad de Ciencias de la Educación. Recuperado de http://helvia.uco.es/xmlui/handle/10396/7194

Manso, M., Pérez, P., Libedinsky, M., Light, D., y Garzón, M. (2011). Las TIC en las aulas: Experiencias latinoamericanas. Buenos Aires: Paidós.

Marín, V. (2004). El conocimiento y la formación del profesorado universitario. Agora Digital, 7. Huelva. Recuperado

www.uhu.es/agora/digital/numeros/07/texto1.htm

Marín, V., y Romero, M. A. (2009). La formación docente universitaria a través de las TICs. Pixel-Bit. Revista de Medios y Educación, 35, 97-103.

Marín, V. (2006). El desarrollo profesional del docente universitario a debate: factores que lo determinan. Universitas Tarraconensis, 29-41 Recuperado de http://pedagogia.fcep.urv.cat/revistaut/revistes/desemb re06aniversari/article02.pdf

Marquès. P. (2007). Innovación educativa con las TIC: Infraestructuras, entornos de trabajo, recursos multimedia, modelos didácticos, competencias TIC. Recuperado http://www.peremarques.net/innovacionescuelaTIC.ht m\#inicio.

Morín, E. (1999). Los siete saberes necesarios para la educación del futuro. Francia: UNESCO.

Pérez, A. (2012). Educarse en la era digital. La escuela educativa. España, Madrid: Morata.

Perrenoud, P. (2004). Diez nuevas competencias para enseñar. Barcelona: Grao.

Prensky, M. (2001). Digital Natives, Digital Inmigrants. On the Horizon, 9,5. Recuperado de http://www.nnstoy.org/download/technology/Digital\% 20Natives\%20-\%20Digital\%20Immigrants.pdf

Ramírez-Martinell, A., y Maldonado, G. A. (2014). Multimodalidad en Educación Superior. En A. Ramírez-Martinell y M. A. Casillas, M.A (Coords.), Háblame de TIC 2 Internet en Educación Superior (pp.19-38). Córdoba: Brujas.
Ramírez-Martinell, A., Casillas, M. A., y Ojeda, M. (2013). Brecha digital entre estudiantes y profesores de la Universidad Veracruzana: Capital cultural; trayectorias escolares y desempeño académico; y grado de apropiación tecnológica. Xalapa, Veracruz, México: Universidad Veracruzana. Recuperado de http://www.uv.mx/blogs/brechadigital/files/2013/08/pr oyecto_brecha_digital_2013_11.pdf

Rojas, I. (2013). Formación y Tecnologías. En Ducoing, P. y Fortoul, B. (Coords.). Procesos de Formación 20022011(pp.479-535). México: ANUIES, Dirección de Medios Editoriales: Consejo Mexicano de Investigación Educativa.

Tascón, T. (2002). Nuevas competencias y roles en la docencia universitaria: La incorporación de las NTICs. En V. Aguilar, J.I. Farray, y J. Brito. Cultura y Educación en la Sociedad de la Información (pp. 287296). A Coruña: Netiblo.

Toffler, A. (1980). La tercera ola. Barcelona: Plaza y Janés Editores.

Torres, S. A., Barona, C., Zúñiga, O.Y., y Soberanes, Y. (2012). TIC y formación del profesorado universitario. En H. Torres y C. Barona (Coords.), Los profesores universitarios y las TIC: Uso, apropiación y experiencias (pp.103-129). México: UAEM: Juan Pablos Editor.

UNESCO (2011). Estándares. Unesco y las iniciativas Intel. Recuperado http://fundacionevolucion.org.ar/sitio/wpcontent/uploads/2013/03/Estandares.Unesco_y_las_iniciativas_Intel.pdf

Universidad Veracruzana. (2014). Dirección General de Desarrollo Académico e Innovación Educativa. Recuperado de http://www.uv.mx/dgdaie/

Zabalza, M. A. (2009). Competencias docentes del profesorado universitario. Calidad y desarrollo profesional. Madrid: Narcea. 